\section{What is meso- zeaxanthin, and where does it come from?}

JM Nolan, K Meagher, S Kashani and S Beatty

\begin{abstract}
The carotenoids lutein (L), zeaxanthin (Z), and meso-zeaxanthin (MZ) accumulate in the central retina, where they are collectively known as macular pigment (MP). Each of these three compounds exhibit a regional dominance, with $M Z, Z$, and $L$ being the dominant carotenoids at the epicentre, mid-periphery, and periphery of the macula, respectively. There is a growing and evidencebased consensus that MP is important for optimal visual performance, because of its blue light-filtering properties and consequential attenuation of chromatic aberration, veiling luminance, and blue haze. It has also been hypothesised that MP may protect against age-related macular degeneration because of the same optical properties and also because of the antioxidant capacity of the three macular carotenoids. Challenges inherent in the separation and quantification of $\mathrm{MZ}$ have resulted in a paucity of data on the content of

zeaxanthin $(\mathrm{Z})$ accumulates at the macula where it is known as macular pigment (MP). ${ }^{2}$

The anatomical (central retinal), ${ }^{2}$ biochemical (antioxidant), ${ }^{3}$ and optical (short-wavelength filtering) ${ }^{4}$ properties of MP have generated interest in the role of MP for vision and macular health. In the first instance, there is an evidencebased consensus that MP is important for vision in normal subjects, ${ }^{5-7}$ which rests on $\mathrm{MP}^{\prime} \mathrm{s}$ ability to optimise visual performance and experience by attenuating chromatic aberration, veiling luminance, and blue haze. ${ }^{8}$

In the second instance, MP has generated interest because of its possible protective role for age-related macular degeneration (AMD), the world's leading cause of age-related blindness. ${ }^{8}$ The protection that MP may offer patients afflicted with, or at risk of, AMD is putatively attributable to its antioxidant properties $^{9,10}$ and/or its pre-receptoral filtration of damaging (short-wavelength) blue light.
\end{abstract} this carotenoid in foodstuffs, and have rendered the study of tissue concentrations of this compound problematic. As a consequence, the few studies that have investigated MZ have, perhaps, been disproportionately influential in the ongoing debate about the origins of this macular carotenoid. Certainly, the narrative that retinal $\mathrm{MZ}$ is derived wholly and solely from retinal $\mathrm{L}$ needs to be revisited. Eye (2013) 27, 899-905; doi:10.1038/eye.2013.98; published online 24 May 2013

Keywords: Meso-zeaxanthin; Lutein; Zeaxanthin; Macular Pigment; Vision

The macular carotenoids and their functions

The central retina, known as the macula, is responsible for optimal spatial vision. ${ }^{1}$ A yellow pigment composed of the carotenoids meso-zeaxanthin (MZ), lutein (L), and

\section{Retinal anatomy of the macular carotenoids}

$\mathrm{MP}$ is at its highest concentration in the receptor axon layer and in the inner plexiform layers, and the concentration of MP peaks at the foveola. ${ }^{11,12}$ $\mathrm{L}$ is the dominant carotenoid in the peripheral macula, $\mathrm{Z}$ in the mid-peripheral macula, and $\mathrm{MZ}$ at the epicentre of the macula. ${ }^{2} \mathrm{~A}$ thorough understanding of the mechanisms governing the selective uptake of MP by the retina has proven to be elusive, although xanthophyll-binding proteins have been identified for $\mathrm{L}^{13}$ and $Z_{,}^{14}$ and $\mathrm{MZ}$ is also known to bind to a Z-binding protein $^{15}$ (study into the nature of the MZbinding protein is currently underway). ${ }^{14}$ Importantly, it has been shown that the Z-binding protein has equal affinity for MZ. ${ }^{15}$

Chemical structure of the macular carotenoids The term carotenoid is used to describe a family of natural organic compounds build from an
Macular Pigment Research Group, Vision Research Centre, Carriganore House, Waterford Institute of Technology, Waterford, Ireland

Correspondence: JM Nolan, Macular Pigment Research Group, Vision Research Centre, Carriganore House, Waterford Institute of Technology, West Campus, Waterford, Ireland. Tel: +353 51834074 ; Fax: +353 872717474 . E-mail: jmnolan@wit.ie

Received: 11 January 2013 Accepted: 23 April 2013 Published online: 24 May 2013 
assembly of isoprenoid units that are important in many aspects of life. Because of their mostly coloured nature and consequential ability to absorb visible light, carotenoids are often referred to as pigments or chromophores. ${ }^{16}$ All carotenoids have a characteristic linear conjugated polyene chain and are classed into one of two subgroups. The hydrocarbon carotenoids are known as carotenes, such as $\beta$-carotene and lycopene, whereas oxygenated derivatives are called xanthophylls, or oxycarotenoids, and include MZ, L, and Z (Figure 1). ${ }^{16}$

The xanthophyll macular carotenoids consist of the typical C40 carotenoid structure, and are substituted with hydroxyl groups at the 3 and $3^{\prime}$ carbon positions. $\mathrm{Z}$ and $\mathrm{MZ}$ both contain two $\beta$-ring end groups, whereas $\mathrm{L}$ contains both a $\beta$-ring and an $\varepsilon$-ring, thereby reducing the level of the conjugation chain length observed in L. In addition, $\mathrm{L}$ has three chiral positions and is therefore described as $3 R, 3^{\prime} R, 6^{\prime} R$ L. $Z$ and $M Z$ are classed as diastereomers, and differ only in the spatial orientation of the hydroxyl group on the C3' chiral position. This chiral position within MZ has an S spatial orientation, whereas $\mathrm{Z}$ has an $\mathrm{R}$ spatial orientation. This difference in spatial arrangement results in $M Z$ being described as $3 R, 3^{\prime} S Z$, and $Z$ being described as $3 R, 3^{\prime} R$ Z. These structural differences of $\mathrm{MZ}, \mathrm{L}$, and $\mathrm{Z}$ have important implications for their respective antioxidant and light-filtering properties. ${ }^{10,17}$

\section{Chemical, light-filtering, and antioxidant properties of the macular carotenoids}

The characteristic vibrant hues of carotenoids are a result of light-absorbing properties provided by their inherent conjugated double-bond system. The resultant UV/vis absorption spectra are routinely used to assist in the identification and characterisation of carotenoids, and have been extensively studied. ${ }^{18,19}$ The absorption spectra of MP peaks at $460 \mathrm{~nm} \cdot{ }^{20}$ Although MZ, L, and Z exhibit very similar absorption spectra, it is possible to distinguish them from one another based on slight variations in relative absorbances $(\mathrm{nm})$ and intensities (AU). ${ }^{21}$

At the macula, $\mathrm{L}$ is reported to be a superior filter of short-wavelength (blue) light when compared with $\mathrm{Z}$, because of its orientation with respect to the plane of the phospholipid bilayer of the cell membrane, which is both parallel and perpendicular. ${ }^{22}$ In contrast, $\mathrm{Z}$ and $\mathrm{MZ}$ exhibit an orientation only perpendicular to this layer. However, structural differences and the consequential differing absorption spectra of these pigments (MZ, L, and $\mathrm{Z}$ ) result in a collective optimal filtration of blue light at the macula, which would not be achieved by any of these carotenoids in isolation. ${ }^{23}$

The polyene chains of $\mathrm{MZ}, \mathrm{L}$, and $\mathrm{Z}$ supply readily available electrons that enable these carotenoids to quench reactive oxygen species (ROS), thus limiting membrane phospholipid peroxidation and attenuating oxidative injury. ${ }^{16,17,22,24,25} \mathrm{Z}$ is twice as efficacious as $\mathrm{L}$ at quenching ROS, and this is attributed to the extended conjugated system of $\mathrm{Z}$ in comparison with L. ${ }^{26}$ This extended conjugation is also present in MZ. When in conjunction with a Z-binding protein, MZ has proven to be a more potent antioxidant than $Z{ }^{14}$ Of interest, a mixture of $\mathrm{L}, \mathrm{Z}$, and $\mathrm{MZ}$ in vitro, in a ratio of $1: 1: 1$, has been shown to quench more singlet oxygen than any of these individual carotenoids at the same total concentration. ${ }^{10}$<smiles>CC1=C[C@H](O)CC(C)(C)C1/C=C/C(C)=C/C=C/C(C)=C/C=C/C=C(C)/C=C/C=C(C)/C=C/C1=C(C)CC(O)CC1(C)C</smiles><smiles>CC1=C(/C=C/C(C)=C/C=C/C(C)=C/C=C/C=C(C)/C=C/C=C(C)/C=C/C2=C(C)C[C@@H](O)CC2(C)C)C(C)(C)CC(O)C1</smiles>

Zeaxanthin<smiles>CO[R4](C)(C)OC(C)=CC=CC=C(C)C=CC=C(C)C=CC=C(C)C=CC1=C(C)C[C@@H](O)CC1(C)C</smiles>

Figure 1 Chemical structures of the three macular carotenoids L, Z, and MZ. 


\section{Identification and quantification of the macular carotenoids}

To quantify MZ, L, and Z, current methodology requires two successive HPLC separations (assays). The first assay (reverse phase chromatography) separates $L$ from a total $\mathrm{Z}$ fraction, which contains $\mathrm{Z}$ and MZ. This total $Z$ fraction is typically manually collected, and then analysed using the second assay (normal phase chromatography). This second assay is required to separate $\mathrm{Z}$ and $\mathrm{MZ}$, so that quantification of these individual carotenoids can be achieved. This general method of carotenoid analysis has been adapted and modified by several laboratories for the purpose of $\mathrm{MZ}$ quantification. ${ }^{21,27-30}$ Modifications are typically made to the first assay, where a variety of reverse phase columns and solvent compositions can produce acceptable $\mathrm{L}$ and total Z separations. ${ }^{31}$ However, the normal phase column (Daicel ChiralPak AD (Daicel Chiral Technologies Europe, Illkirch - Cedex, France); $250 \times 4.6 \mathrm{~mm}^{2}$ ) used in the second assay has remained constant and universal among studies reporting on MZ.

Although the above two-step method is successful at quantifying $\mathrm{MZ}$, it is limited in a number of ways. First, the method is labour intensive because of the requirement to manually collect the total $\mathrm{Z}$ fraction. Also, manual collection is operator-dependent, and therefore vulnerable to human error. As a result, only a portion of the total $\mathrm{Z}$ fraction is collected, and is typically contaminated with L carryover. Second, the relatively long time for sample analysis (in some cases, as much as two hours per sample) makes bulk analysis difficult and costly. Recent developments by our laboratory have included automation of the fraction collection step, which has greatly reduced operator requirements and study analysis time, in addition to improvements in fraction recoveries and elimination of $\mathrm{L}$ carryover. These refinements to the first assay, in combination with advances in HPLC technologies and analytical methodologies, have resulted in better resolution and greater sensitivity for $M Z$ separation and quantification.

\section{Origin of the macular carotenoids}

To date, approximately 700 carotenoids have been isolated and identified in nature, with in excess of 40 found in fruits and vegetables. ${ }^{32,33}$ Despite this, only 14 of these dietary carotenoids may be absorbed, modified, and/or used by the human body, ${ }^{34}$ and yet only MZ, L, and $\mathrm{Z}$ are found at the macula, reflecting an exquisite degree of biological selectivity, which is unlikely to be accidental in design. ${ }^{35}$

Studies have shown that $\mathrm{L}$ and $\mathrm{Z}$ are plentiful in many foodstuffs typical of a western diet (eg, spinach, kale, corn, and egg products). ${ }^{36}$ However, there is a paucity of research investigating $\mathrm{MZ}$ in foodstuffs, apart from a report by Maoka et $a l^{37}$ in 1986, where MZ was confirmed to be present in 21 species of edible fish, shrimp, and sea turtles, and a recent report by Rasmussen et $a l^{21}$ who reported the presence of $\mathrm{MZ}$ in Californian and Mexican hen eggs. Of note, the Mexican hens were given MZ-enriched feed. However, in contrast to the Maoka publication, Rasmussen et $a l^{21}$ did not detect the presence of MZ, L, or Z in any fish (eg, haddock and sea bass) or seafoods (eg, shrimp) analysed in their study.

Importantly, there is a substantial discrepancy between the extraction methods used by Rasmussen et al vs those used by Maoka et al, which merits discussion. Mainly, Rasmussen et al did not saponify the foods before analysis. Saponification is an alkaline hydrolysis (ie, breakdown) of the ester linkages between the carotenoids and lipid (fatty acid) molecules. This is a required step in the process of carotenoid extraction, as it frees esterified carotenoids in the food sample that otherwise would not be observed during analysis, thereby resulting in an underestimation of MZ. Indeed, this point is consistent with and corroborated by Rasmussen et al's own findings, as they identified all three macular carotenoids in egg yolk, because egg carotenoids are not esterified (and therefore the need for saponification is precluded).

It is surprising, therefore, that the view that $\mathrm{MZ}$ is 'non-dietary' in origin has prevailed and gone largely uncontested, given that the MZ content of foodstuffs that contain carotenoids (eg, corn, orange peppers, and yellowtail tuna) has been neither extensively nor satisfactorily investigated.

It had been suggested that the MZ found and reported by Maoka et $a l^{37}$ was just an artefact of degradation from $\mathrm{L}^{21}$ which occurred during the sample-extraction saponification process (given that the biotransformation of $\mathrm{L}$ to $\mathrm{Z}$ is an alkaline hydrolysis reaction). ${ }^{35}$ However, we have tested this possibility in our laboratory and found that saponification of purified $\mathrm{L}$ does not generate $\mathrm{MZ}$, even with high concentrations of $\mathrm{KOH}$. Importantly, for this experiment we purified our L standard via HPLC, as previous work by our laboratory indicated the presence of all $\mathrm{Z}$ isomers (including MZ) in DSMprovided L standards. ${ }^{38}$ Of note, it has also been suggested that MZ may be generated from $L$ as a result of an enzymatic process at the macula, but such a hypothesis has yet to be investigated. ${ }^{28}$

Also, and of interest, a recent investigation of serum carotenoid response following supplementation with different macular carotenoid formulations identified an unexpected peak with the spectrophotometric characteristics of $\mathrm{MZ}$ in the serum of subjects supplemented with $20 \mathrm{mg}$ of L and $2 \mathrm{mg}$ of $\mathrm{Z}$ (Ultra Lutein from Natural Organics Inc., Melville, NY, USA: 
L provided by FloraGLO, which is a registered trademark of Kemin Health, L.C., Kemin Industries, Inc. Des Moines, IA, USA). Based on label claim, these subjects received no MZ. Given that this was an unexpected finding, we reanalysed random serum samples from these subjects in order to confirm or refute our observation, and confirmed that $\mathrm{MZ}$ was, indeed, present. ${ }^{38}$ We then tested the intervention formulation, and determined that it did, in fact, contain MZ (0.3 mg/capsule) (Figure 2), although MZ was not claimed on the product label.

These findings have implications for past and ongoing research surrounding carotenoid supplementation. Indeed, any discrepancy between actual and alleged concentrations of the respective macular carotenoids in commercially available preparations is important when such formulations are used for the purpose of research, particularly clinical trials. Study on the actual vs declared carotenoid content of commercially available supplements is currently being investigated by our group.

Further, our finding that some commercially available $\mathrm{L}$ supplements contain $\mathrm{MZ}$, but where the presence of MZ is neither recognised nor acknowledged in the supplement label, has profound implications for our current understanding of the origins of $\mathrm{MZ}$, as it has been proposed that retinal $\mathrm{MZ}$ is derived solely from retinal $\mathrm{L}$. Of note, this hypothesis is largely driven by the findings of a single DSM-sponsored study, where MZ was identified in the retinae of L-supplemented carotenoid-deprived rhesus monkeys. ${ }^{29}$

This influential study included four groups of six rhesus monkeys. Three groups were maintained on a xanthophyll-free diet, and one group was given a standard diet. Two of the three xanthophyll-free groups were then given xanthophyll supplements. The first were given $\mathrm{L}$ only and the second were given $\mathrm{Z}$ only, whereas the third acted as a control (remaining xanthophyll-free). Two monkeys from each group were killed at 6-10month intervals during the 24-month study period, and their retinae were removed and analysed for carotenoid content. As expected, the control group demonstrated a complete lack of any xanthophyll carotenoid at the macula. The investigators detected $\mathrm{MZ}$ and $\mathrm{L}$ in the central retinae of L-supplemented monkeys, but only $\mathrm{Z}$ in the Z-supplemented primates, and concluded that retinal $\mathrm{MZ}$ was derived wholly from retinal L. ${ }^{29}$ However, we believe that the conclusion by Johnson et $a l^{29}$ cannot and does not follow from their findings, as no study animals were supplemented with $\mathrm{MZ}$, thereby precluding meaningful comment on the bioconversiondependent interrelationships (if any) between the three macular carotenoids.

Further, the recent identification of $\mathrm{MZ}$ in Ultra Lutein, which contains DSM-sourced L, using novel and validated technology, lends credence to the possibility that the L-supplemented rhesus monkeys were in fact being supplemented with small amounts of $\mathrm{MZ}$, thus accounting for the reported concentrations of $\mathrm{MZ}$ detected in the retinae of those animals. Of note, the authors state that the L supplement used in their study was purified L generated for the purpose of their research, although the method of purification was not described and therefore cannot be tested by another laboratory. In addition, it is possible that trace amounts of MZ may not have been detected in the supplement used by the methodologies available at that time. Also, and of concern, an unknown peak was found to co-elute with the $\mathrm{Z}$ fractions of retinal samples in the report by Johnson et $a l^{29}$, which affected accurate quantification of the carotenoids present in these samples. The researchers did make some attempt to address this latter issue by using a

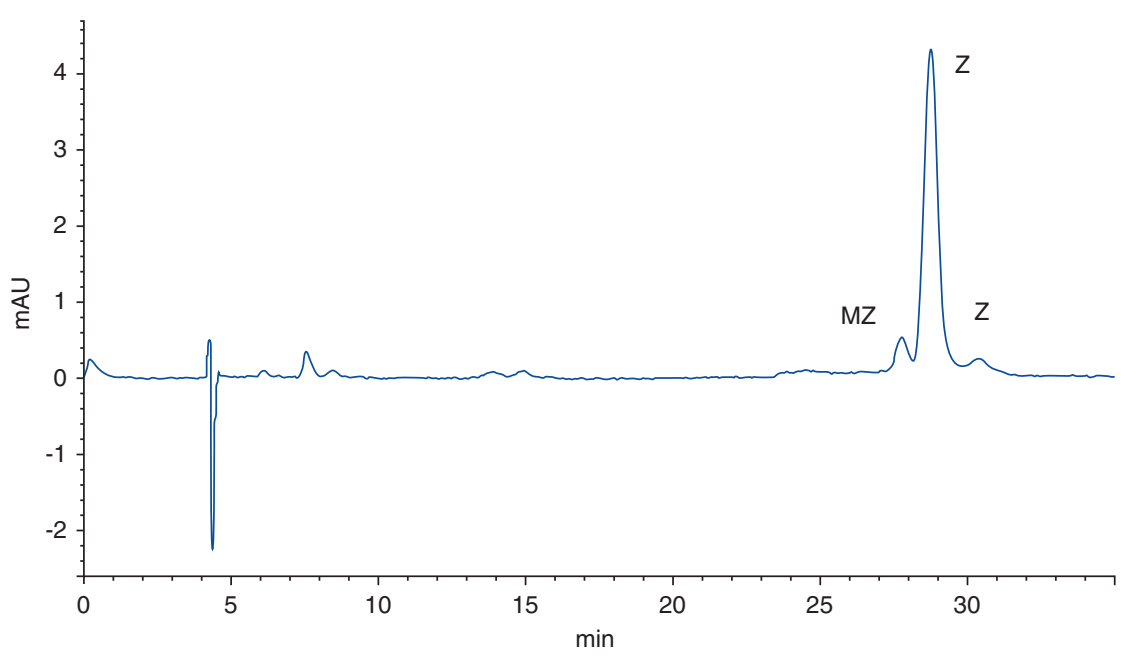

Figure 2 HPLC analysis of the Ultra Lutein supplement. 
method of proportions to identify the contribution of this unknown peak to the $\mathrm{Z}$ fraction. Their method, however, depended on precise and total collection of both $\mathrm{L}$ and $\mathrm{Z}$ fractions (combined) from the first analysis, which, we believe, is impossible to ensure when collecting carotenoid fractions manually (as in their study). Therefore, although the publication reported the presence of $\mathrm{MZ}$ following supplementation, the reported quantities of $\mathrm{MZ}$ and $\mathrm{Z}$ remain questionable. It should also be noted that the researchers did attempt to identify the unknown peak, but conceded that their efforts in this regard were unsuccessful. Accordingly, and in keeping with best scientific practise, we believe that work conducted by Johnson et al ${ }^{29}$ needs to be replicated and extended to include animals supplemented with MZ so that the benefits of recent advances in separation science can be employed in this endeavour.

The hypothesis that retinal MZ is wholly and solely the result of bioconversion of retinal $\mathrm{L}$ is inconsistent with the findings of Bhosale et $a l,{ }^{39}$ who measured deuterated (D)-L, D-Z, and D-MZ in the retinae of quail following supplementation with either D-L, D-Z, or regular diet (control group). After killing, D-L and D-MZ were identified only in animals supplemented with D-L, whereas D-Z was the only isotopically labelled macular carotenoid identified in animals supplemented with D-Z. However, there was a marked discrepancy in the proportion of total retinal L that was deuterated $(83 \%)$ and the proportion of total retinal $\mathrm{MZ}$ that was deuterated (42\%), suggesting that retinal MZ is not derived entirely or exclusively from retinal L.

Of course, it could be postulated that there was a lag in the conversion of supplemented L to MZ, thus explaining the observed discrepancy between the proportions of $\mathrm{MZ}$ and $\mathrm{L}$ that were deuterated, but such a hypothesis requires investigation. Moreover, no study animal was supplemented with isotopically labelled MZ, thereby precluding the opportunity to investigate the possibility of bioconversion of this carotenoid. It is also important to note that the quails were not carotenoid-deficient before supplementation, and therefore the study was not designed to test whether $\mathrm{L}$ is the sole precursor of MZ. Furthermore, the work by Bhosale et al, ${ }^{39}$ should be interpreted with full appreciation of the questionable appropriateness of a non-primate, such as quail, as an animal model for the study of human MP and its origins. ${ }^{31}$

\section{Safety of MZ}

$\mathrm{MZ}$ is now available in commercially available preparations (eg, MacuShield (Macuvision Europe Ltd, Solihull, UK), MacuHealth with LMZ3 (MacuHealth LLC, Birmingham, MI, USA), and Lutein Plus (Holland
\& Barrett, Nuneaton, UK)), and many have been consuming MZ in supplement form since the 1990s, and there are no published accounts of adverse events or safety issues arising from its use. Recently published trials have found that subjects supplemented with all the three macular carotenoids (MZ, L, and Z) exhibit significant increases in serum concentrations of these carotenoids and an associated augmentation in $\mathrm{MP} .{ }^{30,40}$ Indeed, a recent publication reports that the typical central peak of MP can be realised in subjects with atypical MP spatial profiles at baseline when supplemented with a preparation containing all the three macular carotenoids, but not with a supplement lacking $\mathrm{MZ}^{41}$

MZ in such supplements is derived from natural $\mathrm{L}$, which has been extracted from the Aztec Marigold flower, and has been used to date in many clinical trials and subjected to toxicity studies. ${ }^{28,30,40}$ A recent study of supplemental MZ in human subjects has reported that renal and liver function, as well as lipid profile, haematological parameters, and markers of inflammation, are unaffected following supplementation with a formulation containing MZ, $\mathrm{L}$, and $\mathrm{Z} .{ }^{30}$

$\mathrm{MZ}$ is also a component of a typical diet in countries and states where it is commonly used in hen feed to enhance the colouration of the egg yolk by the poultry industry (eg, Mexico), and no associated adverse events have ever been reported.

Moreover, the safety of MZ has been further evaluated in a recent toxicity trial using an animal model (Wister rats). ${ }^{42}$ The results of this trial demonstrated that the NOAEL ('No Observed-Adverse-Effect Level') was in excess of $200 \mathrm{mg} / \mathrm{kg} /$ day, far greater than doses used in dietary supplements, which are typically $<0.5 \mathrm{mg} / \mathrm{kg} /$ day. Absence of mutagenicity was confirmed in the same study, using the Ames test. In 2011, the GRAS ('Generally Regarded As Safe') status of MZ was acknowledged by the FDA in a reply to a proposal from a US company on the status of $\mathrm{MZ}$ (plus L and Z). Finally, a recent safety evaluation of $M Z$ by $\mathrm{Xu}$ et al concluded that MZ has no acute toxicity and no genotoxicity and the use of $\mathrm{MZ}$ is safe at dose of $300 \mathrm{mg} / \mathrm{kg}$ body weight per day in rats from a 90 day feeding study. The authors then applied a 100 fold safety factor, and reported an ADI (acceptable daily intake) of $3 \mathrm{mg} / \mathrm{kg}$ body weight per day for MZ. ${ }^{43}$

\section{Conclusion}

The conviction that retinal $\mathrm{MZ}$ is derived wholly or solely from retinal L is unsafe. Foodstuffs that are likely sources of the macular carotenoids should be 
investigated for the presence of $\mathrm{L}, \mathrm{Z}$, and $\mathrm{MZ}$ using the most modern techniques available to separation science, and any meaningful comment on the bioconversion of these carotenoids within retinal tissue can only be based on the results of supplementation studies that include an intervention arm for each of the three macular carotenoids.

\section{Acknowledgements}

JMN is funded by the European Research Council (ERC).

\section{References}

1 Hirsch J, Curcio CA. The spatial resolution capacity of human foveal retina. Vision Res 1989; 29: 1095-1101.

2 Bone RA, Landrum JT, Friedes LM, Gomez CM, Kilburn MD, Menendez E et al. Distribution of lutein and zeaxanthin stereoisomers in the human retina. Exp Eye Res 1997; 64: 211-218.

3 Khachik F, Bernstein PS, Garland DL. Identification of lutein and zeaxanthin oxidation products in human and monkey retinas. Invest Ophthalmol Vis Sci 1997; 38: 1802-1811.

4 Snodderly DM, Brown PK, Delori FC, Auran JD. The macular pigment.1. absorbance spectra, localization, and discrimination from other yellow pigments in primate retinas. Invest Ophthalmol Vis Sci 1984; 25: 660-673.

5 Nolan JM, Loughman J, Akkali MC, Stack J, Scanlon G, Davison $\mathrm{P}$ et al. The impact of macular pigment augmentation on visual performance in normal subjects: COMPASS. Vision Res 2011; 51(5): 459-469.

6 Stringham JM, Garcia PV, Smith PA, McLin LN, Foutch BK. Macular pigment and visual performance in glare: benefits for photostress recovery, disability glare, and visual discomfort. Invest Ophthalmol Vis Sci 2011; 52: 7406-7415.

7 Walls GL, Judd HD. The intra-ocular colour-filters of vertebrates. Br J Ophthalmol 1933; 17: 705-725.

8 Loughman J, Davison PA, Nolan JM, Akkali MC, Beatty S. Macular pigment and its contribution to visual performance and experience. J Optom 2013; 3: 74-90.

9 Beatty S, Koh HH, Henson D, Boulton M. The role of oxidative stress in the pathogenesis of age-related macular degeneration. Surv Ophthalmol 2000; 45: 115-134.

10 Li B, Ahmed F, Bernstein PS. Studies on the singlet oxygen scavenging mechanism of human macular pigment. Arch Biochem Biophys 2010; 504(1): 56-60.

11 Snodderly DM, Auran JD, Delori FC. The macular pigment II. Spatial distribution in primate retinas. Invest Ophthalmol Vis Sci 1984; 25: 674-685.

12 Trieschmann M, van Kuijk FJ, Alexander R, Hermans P, Luthert $\mathrm{P}$, Bird AC et al. Macular pigment in the human retina: histological evaluation of localization and distribution. Eye (Lond) 2008; 22: 132-137.

13 Li B, Vachali P, Frederick JM, Bernstein PS. Identification of StARD3 as a lutein-binding protein in the macula of the primate retina. Biochemistry 2011; 50(13): 2541-2549.

14 Bhosale P, Bernstein PS. Synergistic effects of zeaxanthin and its binding protein in the prevention of lipid membrane oxidation. Biochim Biophys Acta 2005; 1740: 116-121.

15 Bhosale P, Larson AJ, Frederick JM, Southwick K, Thulin $\mathrm{CD}$, Bernstein PS. Identification and characterization of a pi isoform of glutathione s-transferase (GSTP1) as a zeaxanthin-binding protein in the macula of the human eye. J Biol Chem 2004; 279: 49447-49454.

16 Britton G. Structure and properties of carotenoids in relation to function. FASEB J 1995; 9: 1551-1558.

17 Pintea A, Rugina D, Pop R, Bunea A, Socaciu C. Xanthophylls protect against induced oxidation in cultured human retinal pigment epithelial cells. J Food Composition Anal 2011; 24: 830-836.

18 Britton G. UV/visible spectroscopy. In: Britton G, LiaaenJensen S, Pfander H (eds). Carotenoids: Spectroscopy Birkhäuser, Basel, Switzerland; Boston, MA; Berlin, Germany, 1995; Vol. 1B, pp 13-63.

19 Davies BH. Carotenoids. In: Goodwin TW (eds). Chemistry and Biochemistry of Plant Pigments. Academic Press: London, UK, 1976; Vol. 2, pp 38-165.

20 Pease PL, Adams AJ, Nuccio E. Optical-density of human macular pigment. Vision Res 1987; 27: 705-710.

21 Rasmussen H, Muzhingi T, Eggert T, Johnson EJ. Lutein, zeaxanthin, meso-zeaxanthin content in egg yolk and their absence in fish and seafood. J Food Composition Anal 2012; 27: 139-144.

22 Sujak A, Gabrielska J, Grudzinski W, Borc R, Mazurek P, Gruszecki WI. Lutein and zeaxanthin as protectors of lipid membranes against oxidative damage: The structural aspects. Arch Biochem Biophys 1999; 371: 301-307.

23 Billsten HH, Bhosale P, Yemelyanov A, Bernstein PS, Polivka T. Photophysical properties of xanthophylls in carotenoproteins from human retinas. Photochem Photobiol 2003; 78: 138-145.

24 Trevithick-Sutton CC, Foote CS, Collins M, Trevithick JR. The retinal carotenoids zeaxanthin and lutein scavenge superoxide and hydroxyl radicals: a chemiluminescence and ESR study. Mol Vis 2006; 12: 1127-1135.

25 Junghans A, Sies H, Stahl W. Macular pigments lutein and zeaxanthin as blue light filters studied in liposomes. Arch Biochem Biophys 2001; 391: 160-164.

26 Cantrell A, McGarvey DJ, Truscott TG, Rancan F, Bohm F. Singlet oxygen quenching by dietary carotenoids in a model membrane environment. Arch Biochem Biophys 2003; 412 $47-54$.

27 Thurnham DI, Tremel A, Howard AN. A supplementation study in human subjects with a combination of mesozeaxanthin, $\left(3 R, 3^{\prime} R\right)$-zeaxanthin and $\left(3 R, 3^{\prime} R, 6^{\prime} R\right)$-lutein. Br J Nutr 2008; 100: 1307-1314.

28 Bone RA, Landrum JT, Cao Y, Howard AN, varez-Calderon F. Macular pigment response to a supplement containing meso-zeaxanthin, lutein and zeaxanthin. Nutr Metab (Lond) 2007; 4(12): 12.

29 Johnson EJ, Neuringer M, Russell RM, Schalch W, Snodderly DM. Nutritional manipulation of primate retinas, III: effects of lutein or zeaxanthin supplementation on adipose tissue and retina of xanthophyll-free monkeys. Invest Ophthalmol Vis Sci 2005; 46: 692-702.

30 Connolly EE, Beatty S, Loughman J, Howard AN, Louw MS, Nolan JM. Supplementation with all three macular carotenoids: response, stability, and safety. Invest Ophthalmol Vis Sci 2011; 52: 9207-9217.

31 Khachik F, de Moura FF, Zhao DY, Aebischer CP, Bernstein PS. Transformations of selected carotenoids in plasma, liver, and ocular tissues of humans and in nonprimate animal models. Invest Ophthalmol Vis Sci 2002; 43: 3383-3392.

32 Khachik F, Beecher GR, Goli MB. Separation, identification and quantification of carotenoids in fruits, vegetables and 
human plasma by high performance liquid chromatography. Pure Appl Chem 1991; 63: 71-80.

33 Khachik F, Beecher GR, Goli MB, Lusby WR. Separation and quantitation of carotenoids in foods. Methods Enzymol 1992; 213(347-59): 347-359.

34 Khachik F, Beecher GR, Goli MB. Separation and identification of carotenoids of carotenoids and their oxidation products in the extracts of human plasma. Anal Chem 1992; 64: 2111-2122.

35 Bone RA, Landrum JT, Hime GW, Cains A, Zamor J. Stereochemistry of the Human Macular Carotenoids. Invest Ophthalmol Vis Sci 1993; 34: 2033-2040.

36 Perry AF, Rasmussen HM, Johnson EJ. Xanthophyll (lutein, zeaxanthin) content in fruits, vegetables and corn and egg products. J Food Composition Anal 2009; 22: 9-15.

37 Maoka T, Arai A, Shimizu M, Matsuno T. The first isolation of enantiomeric and meso-zeaxanthin in nature. Comp Biochem Physiol B 1986; 83: 121-124.

38 Meagher KA, Thurnham DI, Beatty S, Howard AN, Connolly E, Cummins W et al. Serum response to supplemental macular carotenoids in subjects with and without age-related macular degeneration. Br J Nutr 2012; $1-12$.

39 Bhosale P, Serban B, Zhao dY, Bernstein PS. Identification and metabolic transformations of carotenoids in ocular tissues of the Japanese quail Coturnix japonica. Biochemistry 2007; 46: 9050-9057.

40 Connolly EE, Beatty S, Thurnham DI, Loughman J, Howard AN, Stack J et al. Augmentation of macular pigment following supplementation with all three macular carotenoids: an exploratory study. Curr Eye Res 2010; 35 335-351.

41 Nolan JM, Akkali MC, Loughman J, Howard AN, Beatty S. Macular carotenoid supplementation in subjects with atypical spatial profiles of macular pigment. Exp Eye Res 2012; 101: 9-15.

42 Chang CJG. Thirteen week oral (gavage) toxicity of meso-zeaxanthin in Han Wister rats with a 4-week recovery. Gene Logic Laboratories, Inc.: Gaithersburg, MD, 2006, Study no. 1567-04370.

43 Xu X, Zhang L, Shao B, Sun X, Ho CT, Li S. Safety evaluation of meso-zeaxanthin. Food Control 2013; 32: 678-686. 